\title{
GCU
}

Glasgow Caledonian

University

University for the Common Good

\section{Autonomous rolling-stock coupler inspection using industrial robots}

Vithanage, Randika K. W.; Harrison, Colin S.; De Silva, Anjali K.M.

Published in:

Robotics and Computer-Integrated Manufacturing

DOI:

10.1016/j.rcim.2019.03.009

Publication date:

2019

Document Version

Author accepted manuscript

Link to publication in ResearchOnline

Citation for published version (Harvard):

Vithanage, RKW, Harrison, CS \& De Silva, AKM 2019, 'Autonomous rolling-stock coupler inspection using industrial robots', Robotics and Computer-Integrated Manufacturing, vol. 59, pp. 82-91.

https://doi.org/10.1016/j.rcim.2019.03.009

\section{General rights}

Copyright and moral rights for the publications made accessible in the public portal are retained by the authors and/or other copyright owners and it is a condition of accessing publications that users recognise and abide by the legal requirements associated with these rights.

Take down policy

If you believe that this document breaches copyright please view our takedown policy at https://edshare.gcu.ac.uk/id/eprint/5179 for details of how to contact us. 


\section{Autonomous rolling-stock coupler inspection using industrial}

\section{robots}

Randika K. W. Vithanage, Colin S. Harrison and Anjali K.M. De Silva

School of Engineering and Built Environment, Glasgow Caledonian University, Cowcaddens Road, Glasgow G4 OBA, United Kingdom

In railway rolling-stock maintenance, where the environment is highly unstructured and unpredictable, the detection and localization of smaller components is difficult and remains a key challenge in introducing autonomous systems. This paper presents the design and evaluation of a system, which allows industrial robots to inspect the electric head of railway couplers. The system developed comprising multiple sensors and active-compliant end of arm tooling, is robust to noise and ambiguities. Successful validation of the system is achieved using both an actual coupler in its working environment and an experimental setup that includes a commercial grade industrial robot and a mock coupler.

Key words: 3D-Image processing, Force-control, Sensor fusion, autonomous maintenance

\section{Introduction}

Rolling stock maintenance is a crucial part of the railway industry. The current practice however, involves large expenditure, exposure of workers to hostile conditions, and prone to inconsistencies and inaccuracies [1]. Robotic and autonomous systems (RAS), have the potential to yield reduced costs, improved quality, efficiency and repeatability [2], and thus would be an ideal solution to overcome the challenges in railway maintenance [3]. Although the application of robotics in manufacturing industry is well researched/documented, the opportunities in railway maintenance domain are yet to be explored in depth. This paper presents a specific example of a maintenance task and addresses some of the key aspects of a practical implementation. The unpredictable nature of maintenance tasks and partially organised environments such as railway maintenance depots can present issues for the deployment of RAS and demands advanced sensing techniques, decision making and processing capabilities [4].

\subsection{Scope of the study and research challenge}

The core objective of this study is to present a low cost retrofit solution to automate the electric continuity test of Scharfenberg type railway couplers attached SIEMENS Class 380 rolling-stock using a six degree of freedom (DoF) commercially available articulated industrial robot - shown in figure 1 (a) and (b). The 
system consists of a unified 3D vision and force sensing scheme and a prototype end of arm tooling (EOAT).

(a)

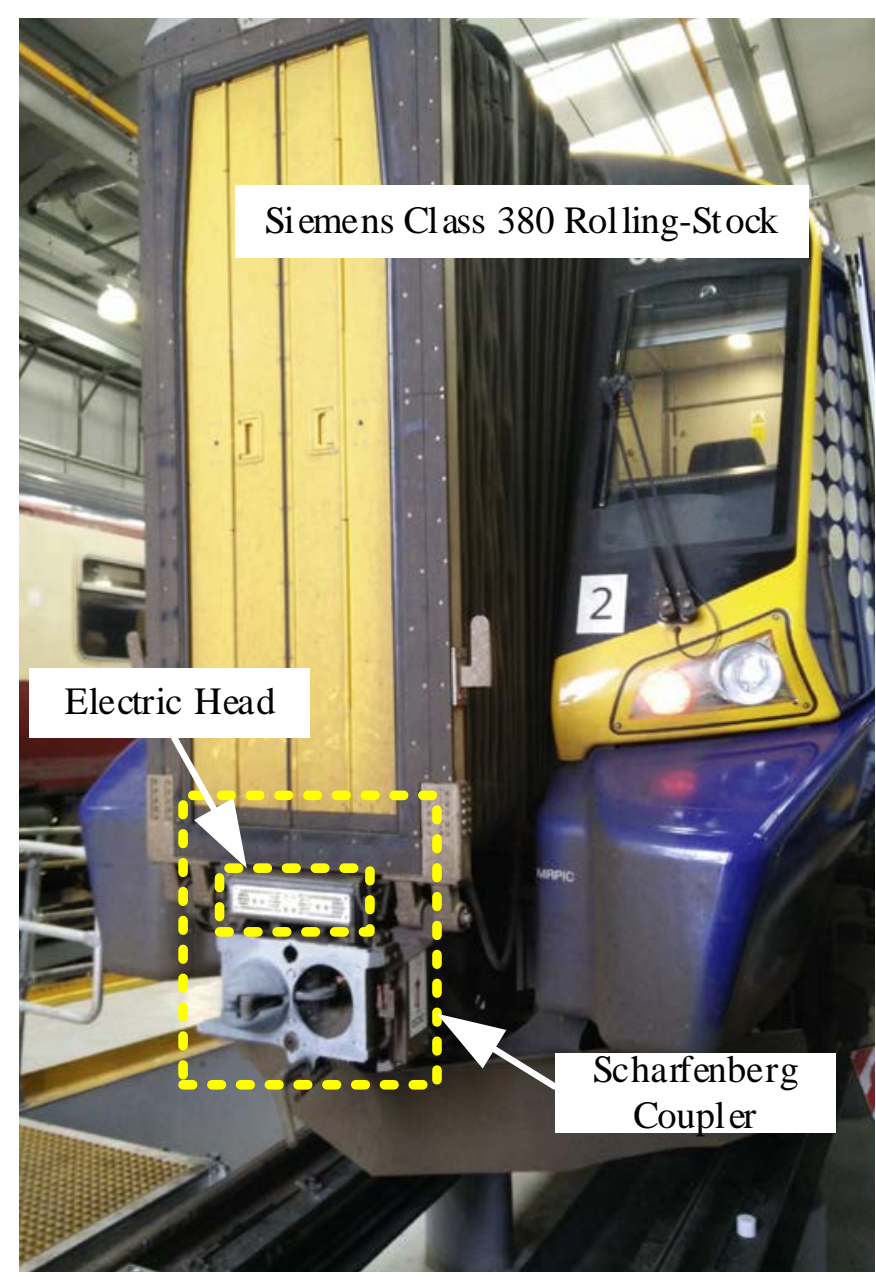

(b)

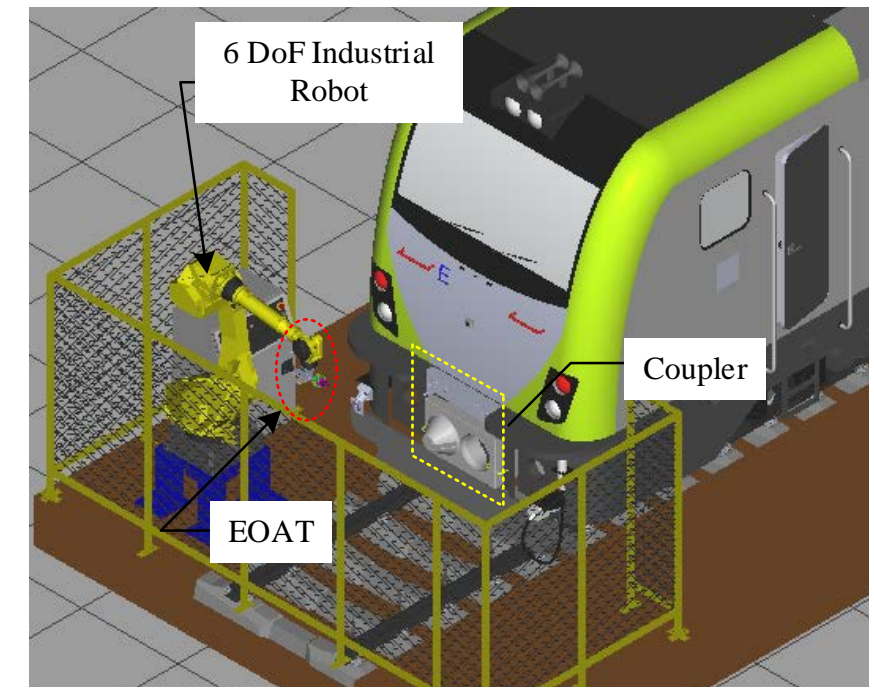

(c)

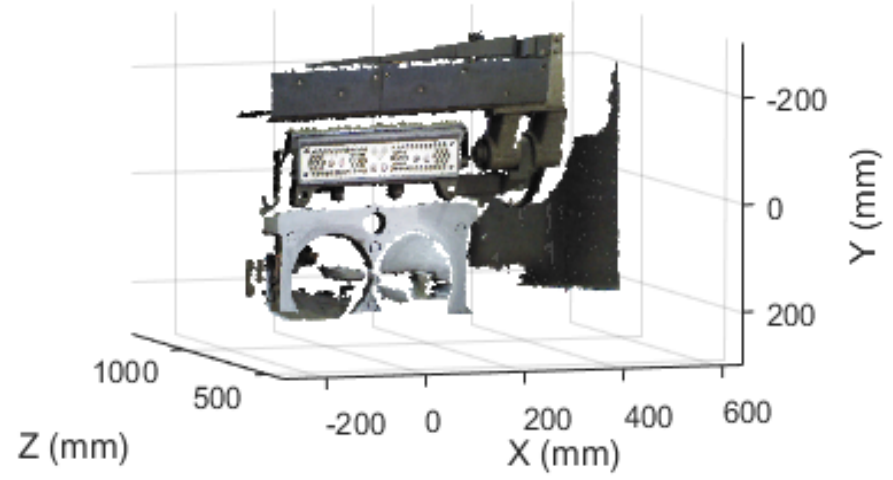

Figure 1. (a) Scharfenberg type railway coupler, (b) conceptual robotic work cell, and (c) 3D point cloud of the coupler.

Timely inspection of the electric head, albeit frequent and monotonous, is critically important as it provides the required electrical coupling between rolling stock. The overall inspection procedure includes general and detailed cleaning, visual inspection, and electrical continuity tests. The whole process consumes up to 30 minutes per operation. The overall process might appear trivial for skilled operators but offers interesting challenges for a RAS. Such challenges include, but are not limited to identification and localization of relatively smaller targets in an uncalibrated environment. For instance, the precise control of train's stopping distance, or the pose of the coupler itself which has the freedom to move in 3D space is not feasible. Similarly, it can be challenging to regulate other environmental factors such as the amount of dirt, contrast of paint, and treatments done to the coupler facet. Hardly any published research work can be found on unified 3D visual servoing and force-position control scheme for rolling-stock maintenance, or on automating rolling-stock front-end inspection. 


\subsection{Related work, 3D robotic vision and force control}

At present, there is growing interest in automating maintenance and inspection tasks. In [5], a scalable hexapod robots has been proposed for maintenance, repair and operations within remote, irregular and hazardous environments. Further, in [6], an intelligent automation scheme was proposed for predictive maintenance where the researchers have used Neural Networks and Bayesian Networks to monitor and diagnosis of non-critical machinery. A tethered mobile robot for highway maintenance was described in [7]. In here a self-propelled robot with position tracking and differential steering technique was introduced. The core objective of the said robot is to support maintenance vehicles with power, materials and etc. A remote condition based maintenance scheme for robots has introduced in [8] where a remotely located maintenance personnel is able to monitor the condition of harmonic drives attached to each axis and take timely decisions regarding their replacement. A new software for 6-DOF articulated robot manipulators was introduced in [9]. The MATALB ${ }^{\circledR}$ toolbox described in here enables flexible trajectory planning for the non-destructive testing of surfaces often found in engineering production.

(a)

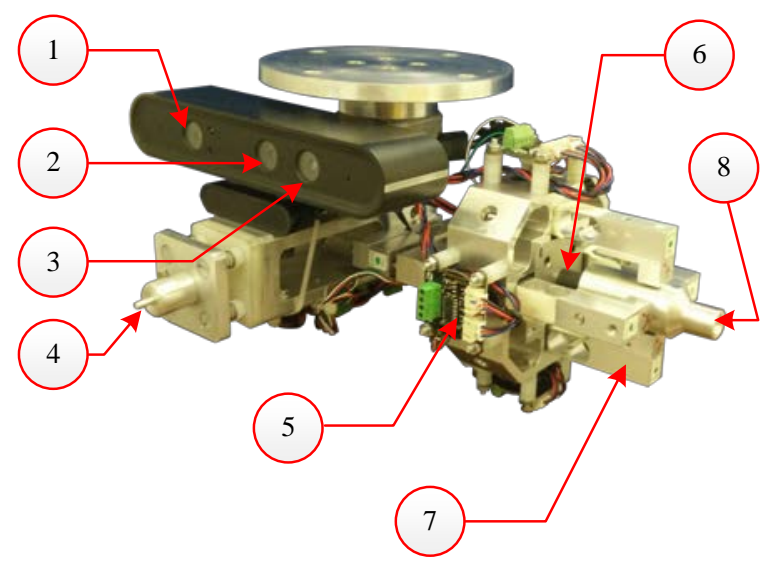

(b)

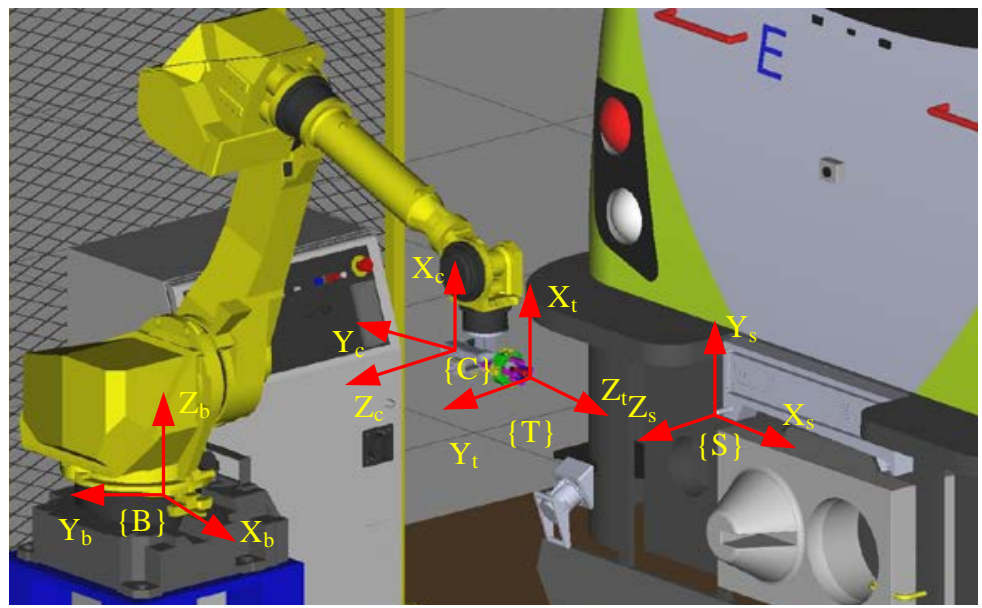

Figure 2. (a) EOAT: (1) IR projector, (2) RGB Camera, (3) IR receiver, (4) Tool 2, (5) voltage amplifier and A/D converter x 6 , (6) flex mount, (7) force transducer x 6 and (8) Tool 1, and (b) reference coordinate frames: $\{S\}$ coupler coordinate frame, $\{C\}$ RGB camera coordinate frame, $\{\mathrm{T}\}$ tool frame and $\{\mathrm{B}\}$ robot's base frame.

The 3D vision is one of key perception techniques used in industrial robotics to locate, inspect and process objects [10]. Such applications include 2D and 3D sensors. As shown is figure 1 (c) valuable features of the target scene can be reconstructed using 3D vision. Here, a RGB camera combined with a time of flight (ToF) camera at $640 \times 480$ resolution was used. A robotic work cell and a calibration methodology has been introduced using ToF 3D point cloud data and prior known geometric information of the system in [11]. Further, in [12] a low cost 3D sensor which comprises an RGB sensor and a ToF sensor was successfully adapted to a manufacturing application with a focus on robotics. An indoor robotic inspection system which utilizes 3D vision is described in [13]. Furthermore, a programming-free, remote robotic 
assembly system is presented in where the system generates 3D models of the unknown parts by taking multiple images with a calibrated 2D camera and fit them to the geometric model of the robotic cell allowing a remote operator to manipulate the robot.

Similarly, crucial information about the manipulator's interaction with its surroundings can be estimated by measuring the forces and torques exerted at the end effector. This information is used, not only to control the forces applied by robot on its environment, but also to position the robot or to make the robot's end effector compliant in a given direction. In [14], a force-controlled end effector for polishing is presented. The proposed low-inertial tool is capable of achieving commendable force tracking with a reduced vibration of the system. A prototype tool for plastic cantilever snap-fit battery covers is presented in [15]. In here, the force sensitive resistors were used to measure the applied force on the tool tip. The system was tested on multiple disassembly tasks using a Cartesian robot where researchers have obtained successful results.

Nevertheless, both vision and force-torque sensing systems that are used to position robots, have inherent drawbacks. For instance, vision systems are capable of global target localization and perform well in calibrated environments. However, they can suffer from inaccuracies when deployed in unstructured environments. On the other hand, force-position control systems are good at providing precise information on the target and less prone to environmental noise in uncalibrated environments, but lack global target localization capabilities. This has led to various approaches to combine both visual and force-torque servoing techniques as described in [16].

\section{System overview}

A traded servoing`mechanism was used to control the robot where manipulation is alternatively controlled by 3D vision and force. The physical system comprises a custom designed end of arm tooling (EOAT), an RGB camera and a ToF camera in eye-in-hand configuration, six force transducers: each coupled to a voltage amplifier and a 24 bit A/D converter, and an ATmega2560 microcontroller, shown in figure 2 (a). The coordinates frames depicted in figure 2 (b) will be used hereafter to describe kinematic transformation of the system. The software portion of the system is designed using MATLAB ${ }^{\circledR}$ and Simulink ${ }^{\mathrm{TM}}$ software packages as a standalone application. Prior to implementation, both RGB and ToF cameras were calibrated, and the tool centre point (TCP) and hand-eye calibration was performed. The overall system is configured in four stages as shown in figure 3: Stage (1) a visual servoing module for orientation (VSOM), Stage (2) a visual servoing module for position (VSPM), Stage (3) a force-position control module (FPCM) and Stage (4) a voltage sensing module (VSM). It has been assumed that the position of the target is static throughout the inspection process. 


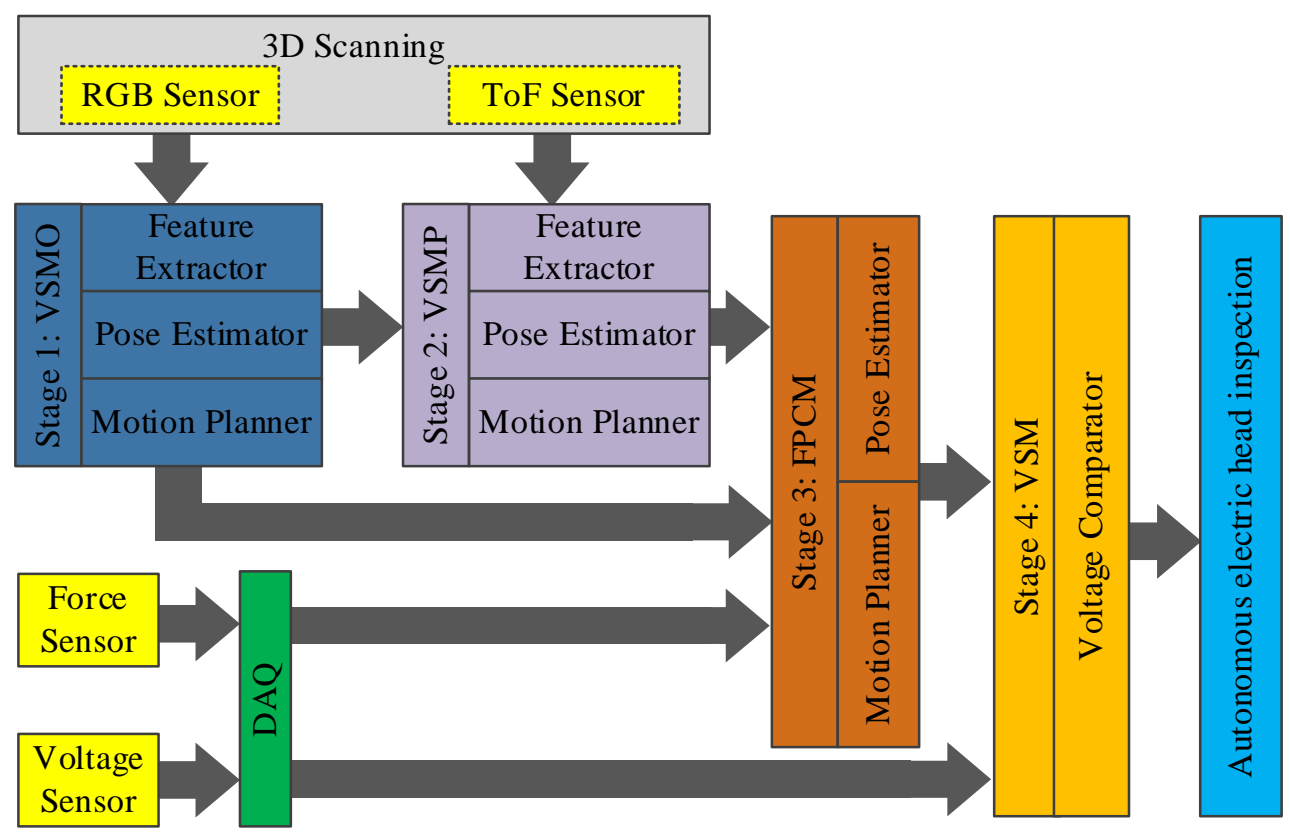

Figure 3: Overall workflow of the system

The complete process is fourfold. In the initial stage, the open loop VSOM manipulates the robot such that $Z_{t}$ is parallel to $Z_{s}$ (normalized TCP). In this instance, the rotation around $Z_{t}$ is neglected exploiting the advantage of electrical pin geometry. In the $2^{\text {nd }}$ stage, the open loop VSPM estimates an approach position for the robot which will be the input to FPCM. In the $3^{\text {rd }}$ stage, the FPCM manipulates robot in a closed loop such that the desired engagement between a pin and the EOAT is achieved. In the final stage, VSM compares the voltage for a given pin. The $1^{\text {st }}$ and $2^{\text {nd }}$ stages are onetime operations while the $3^{\text {rd }}$ and $4^{\text {th }}$ stages are iterative for each pin.

\subsection{Visual servoing module for orientation (VSOM)}

The feature extractor (FE) of VSOM identifies the coupler electric head facet. To enhance useful features such as edges, the input image $I_{S}$ was first sharpened using an un-sharp masking and then a self-guided filter was applied iteratively to reduce the noise while preserving edges. As shown in the figure 4 (a)-(c), the outer edges of the coupler were detected using the "Canny" edge detector. The isolated edges were further optimized by performing several morphological operations the edge image $E_{s}$ was generated such that only the polygon (Poly) which represents the bounding box of the coupler electric head remains. The isolated Poly boundary are illustrated in figure 4 (d) to (f). 
(a)

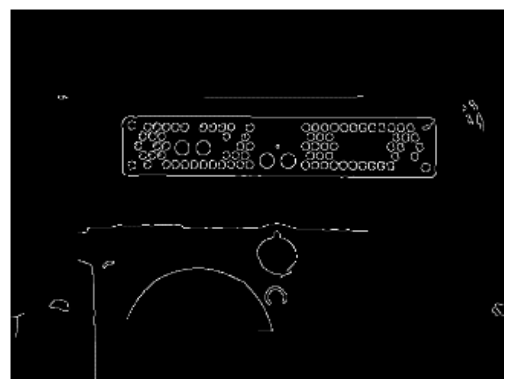

(d)

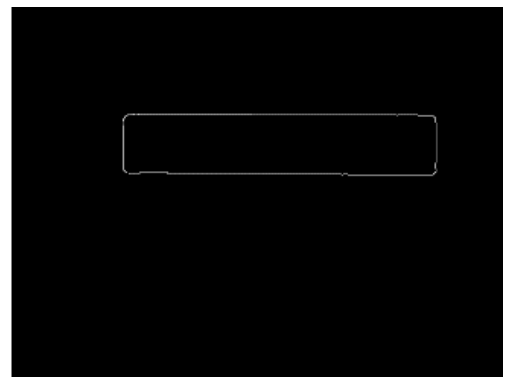

(g)

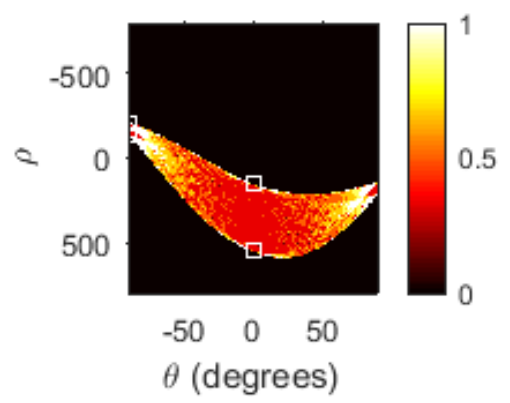

(j)

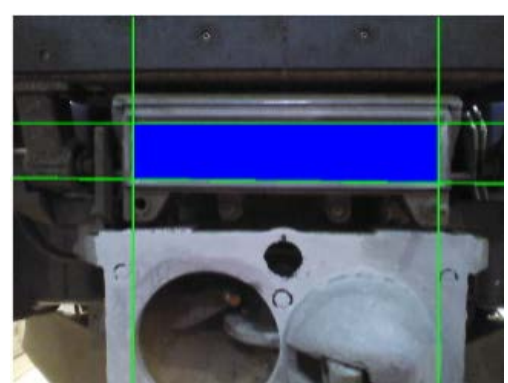

(b)

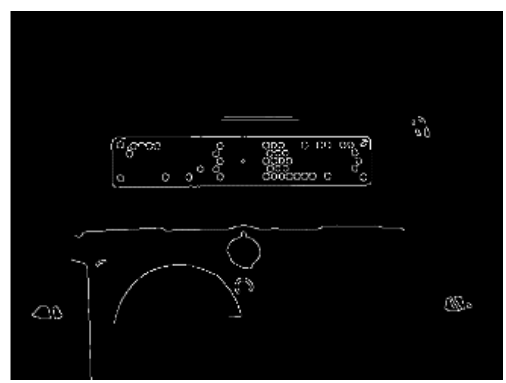

(e)

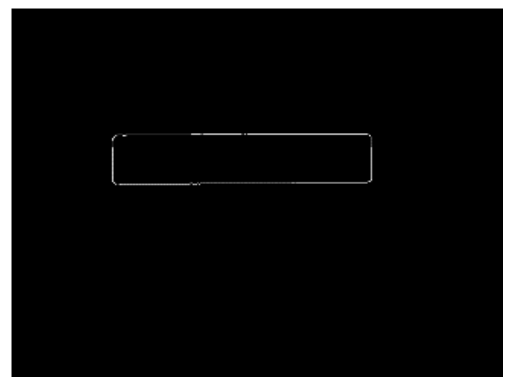

(h)

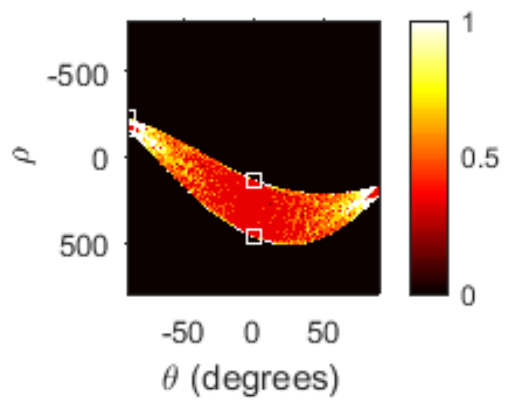

(k)

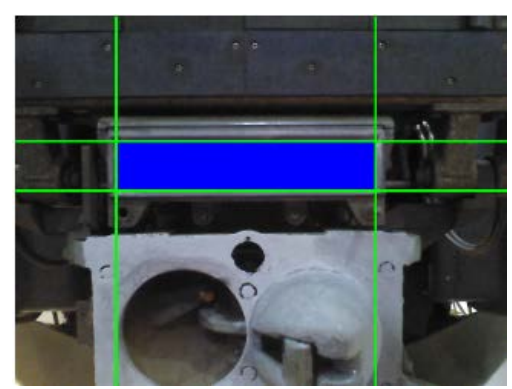

(c)

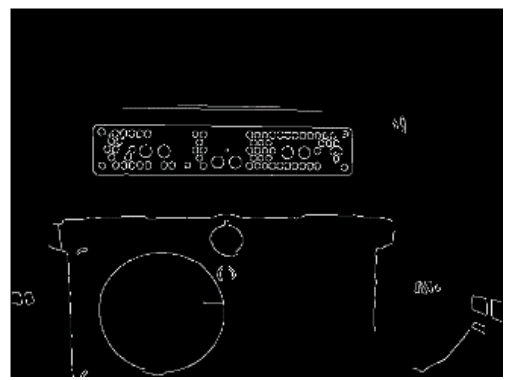

(f)

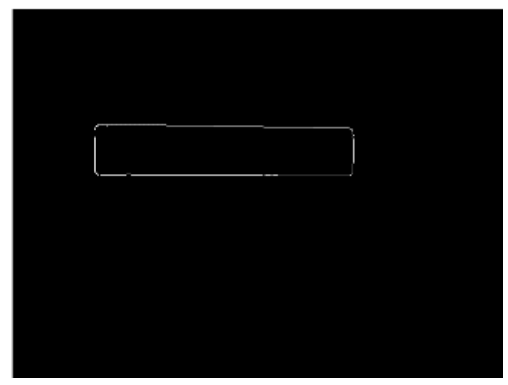

(i)

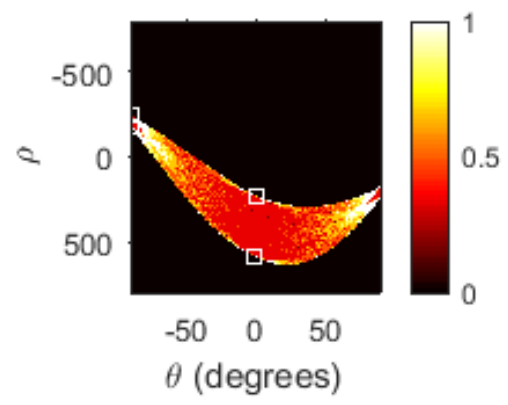

(l)

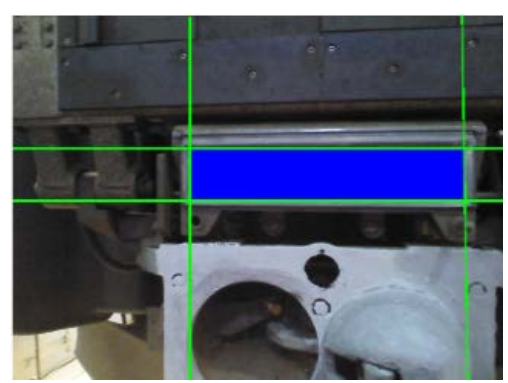

Figure 4

A parametric equation of a line can be written as $\rho=x \cos \theta+y \sin \theta$ where $\rho$ is the shortest distance from origin to the line and $\theta \in[0, \pi]$ is the clockwise angle between $X$ axis of a right handed coordinate system and the perpendicular from origin to line. Given a single edge pixel on the image $E_{S}$, then the set of all lines go through that point correspond to a sinusoidal curve in the $(\rho, \theta)$ plane - which is unique to that point. A set of two or more edge pixels that form a straight line will produce sinusoidal curves that cross each other on $(\rho, \theta)$ plane. Therefore, it is viable to detect the $\rho$ and $\theta$ parameters of the straight lines which 
represent four edges of the polygon by finding the four points with maximum overlaps in $(\rho, \theta)$ space. Shown in figure $4(\mathrm{j})-(\mathrm{l})$ are the estimated lines projected on to the respective $I_{s}$. The pixels belongs to the Poly were determined with the help of MATLAB ${ }^{\circledR}$ Mapping Toolbox ${ }^{\mathrm{TM}}$ which closely performs an inpolygon test proposed by [17]. All pixels $p_{i} \in$ Poly were then determined and summarized to label matrix $L$ - which subsequently used by VSMO pose estimator module as one of its inputs. Refer figure 4 (j)-(l) for the visualization of detected coupler facet which is projected on the individual $I_{S}$.

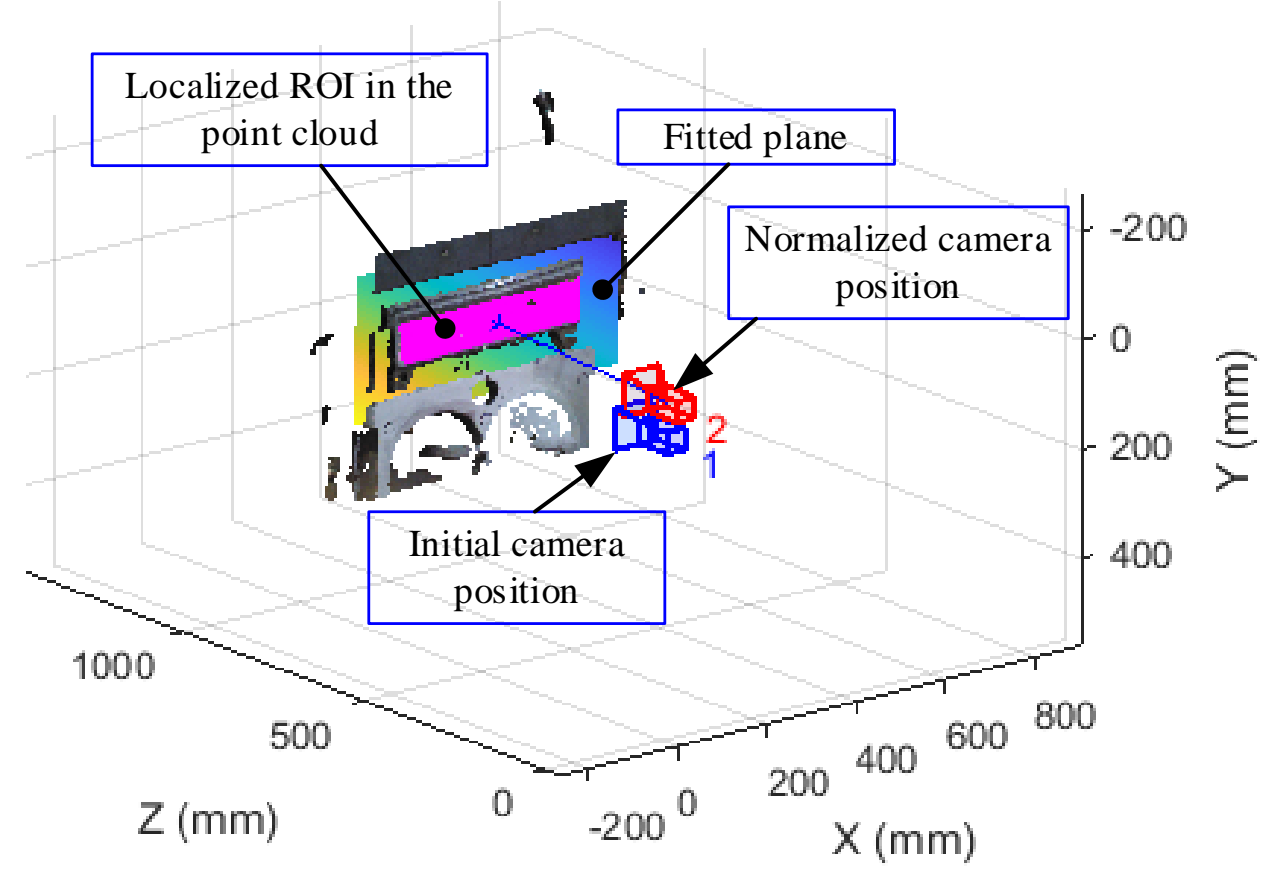

Figure 5: Estimated couple facet model and normalized camera pose

The VSOM pose estimator (PE) generates a 3D point cloud of the scene in RGB camera frame. In general, ToF sensors are prone to noise. Therefore, a Generalized Extreme Studentized Deviation (GESD) test was performed to remove the outliers. By knowing the 3D geometry of the coupler electric head, it is viable to estimate its pose in robot's space. Therefore, the geometric model of the electric head facet is assumed to be a plane and computed by employing the principal component analysis (PCA) ordination. The axis with the maximum moment of inertia of the point nodes is taken as the vector normal to the best-fit plane. Further, the best-fit plane contains the centroid of the point cloud. Therefore, the 3D point cloud data belongs to coupler front plate is normalized by subtracting the centroid $(\bar{X}, \bar{Y}, \bar{Z})$ or the origin of the system. The matrix $\mathrm{M}$ is defined as illustrated in equation 1 where $X_{i}{ }^{\prime}, Y_{i}{ }^{\prime}$ and $Z_{i}{ }^{\prime}$ are the normalized point coordinates. The matrix $\mathrm{M}$ is the covariance matrix of 3D point cloud data and therefore, by realizing $|M-\lambda I|=0$ and $[M-\lambda I] v=0$, the Eigen values $\left(\lambda_{1}, \lambda_{2}, \lambda_{3}\right)$ and Eigen vector $(v)$ of the covariance matrix was calculated where $I$ is a $3 \mathrm{X} 3$ identity matrix. The eigenvector associated with smallest eigenvalue represents the axis with maximum inertia or the vector normal to the best-fit plane. 
Equation 1

$M=\left[\begin{array}{ccc}\sum x_{i}^{\prime 2} & \sum x_{i}^{\prime} y_{i}^{\prime} & \sum x_{i}^{\prime} z_{i}^{\prime} \\ \sum x_{i}^{\prime} y_{i}^{\prime} & \sum y_{i}^{\prime 2} & \sum y_{i}^{\prime} z_{i}^{\prime} \\ \sum x_{i}^{\prime} z_{i}^{\prime} & \sum y_{i}^{i} z_{i}^{\prime} & \sum z_{i}^{\prime 2}\end{array}\right]$

The kinematic transformation for normalized camera pose with respect to the target $\left({ }_{S}^{C} T\right)$ is derived using equation 2 where, $\underline{\hat{n}}$ is the unit vector perpendicular to the best-fit plane, $\theta$ is the rotation about axis $X_{c}$ given by $\cos ^{-1}(\underline{\hat{n}} \cdot \underline{j}), \psi$ is rotation about axis $Y_{c}$ given by $\cos ^{-1}(\underline{\hat{n}} \cdot \underline{i}), x_{n}, y_{n}$ and $z_{n}$ denote the 3D coordinates of the point where a line which passes through the centroid of plane in the direction of $\underline{\hat{n}}$ intersects $X_{c} Y_{c}$ plane, and $\underline{\mathrm{i}} \mathrm{j}$, are the unit vectors along $X_{c}$ and $Y_{c}$ respectively. Finally, the motion planner calculates the transformation matrix between tool and coupler $\left({ }_{S}^{T} T\right)$ according to ${ }_{S}^{T} T={ }_{C}^{T} T \times{ }_{S}^{C} T$ where ${ }_{C}^{T} T$ is the transformation from frame $\{\mathrm{T}\}$ to $\{\mathrm{C}\}$ which is realized through hand-eye calibration and ${ }_{S}^{C} T$ is the transformation from frame $\{C\}$ to $\{S\}$. Shown in figure 5 is the $3 D$ reconstruction of the target in RGB camera frame, localized spatial point belong to the coupler facet, estimated plane, initial camera pose and the normalized camera pose.

Equation 2

${ }_{S}^{C} T=\left[\begin{array}{cccc}\cos \psi & 0 & \sin \psi & x_{n} \\ \sin \theta \sin \psi & \cos \theta & -\sin \theta \cos \psi & y_{n} \\ -\cos \theta \sin \psi & \sin \theta & \cos \theta \cos \psi & z_{n} \\ 0 & 0 & 0 & 1\end{array}\right]$

\subsection{Visual servoing module for position (VSPM)}

The core objective of the VSPM FE is to identify the electrical connector pin candidates so that the tool can be correctly aligned to test the connectors. Due to their size, shape, surface texture and environmental noise, it is nontrivial to identify and localize these connectors through conventional edge detection methodologies. To this end, a lookup table combined with feature mapping is proposed to detect and localize the connector pins. Two precompiled databases are created encapsulating intensity and prior known coupler geometry data for both male and female electrical connector pins. The overall workflow of the module is depicted in figure 6. A normalized 2D cross-correlation coefficient analysis is performed according to equation 3 where $f$ is the target image, $t$ is the template image with dimensions $u$ and $v$ placed at $(x, y)$ on the target image, $\bar{f}_{\mathrm{u}, \mathrm{v}}$ is the mean of $f$ under the region covered by template, $\bar{t}$ is the mean of feature. The best matched templates are identified as the ones that provides highest $C_{c}$. 
Equation 3

$C_{c}=\frac{\sum_{x y}\left[f(x, y)-\bar{f}_{u, v}\right][t(x-u, y-v)-\bar{t}]}{\left\{\sum_{x y}\left[f(x, y)-\bar{f}_{u, v}\right]^{2} \sum_{x y}[t(x-u, y-v)-\bar{t}]^{2}\right\}^{0.5}}$

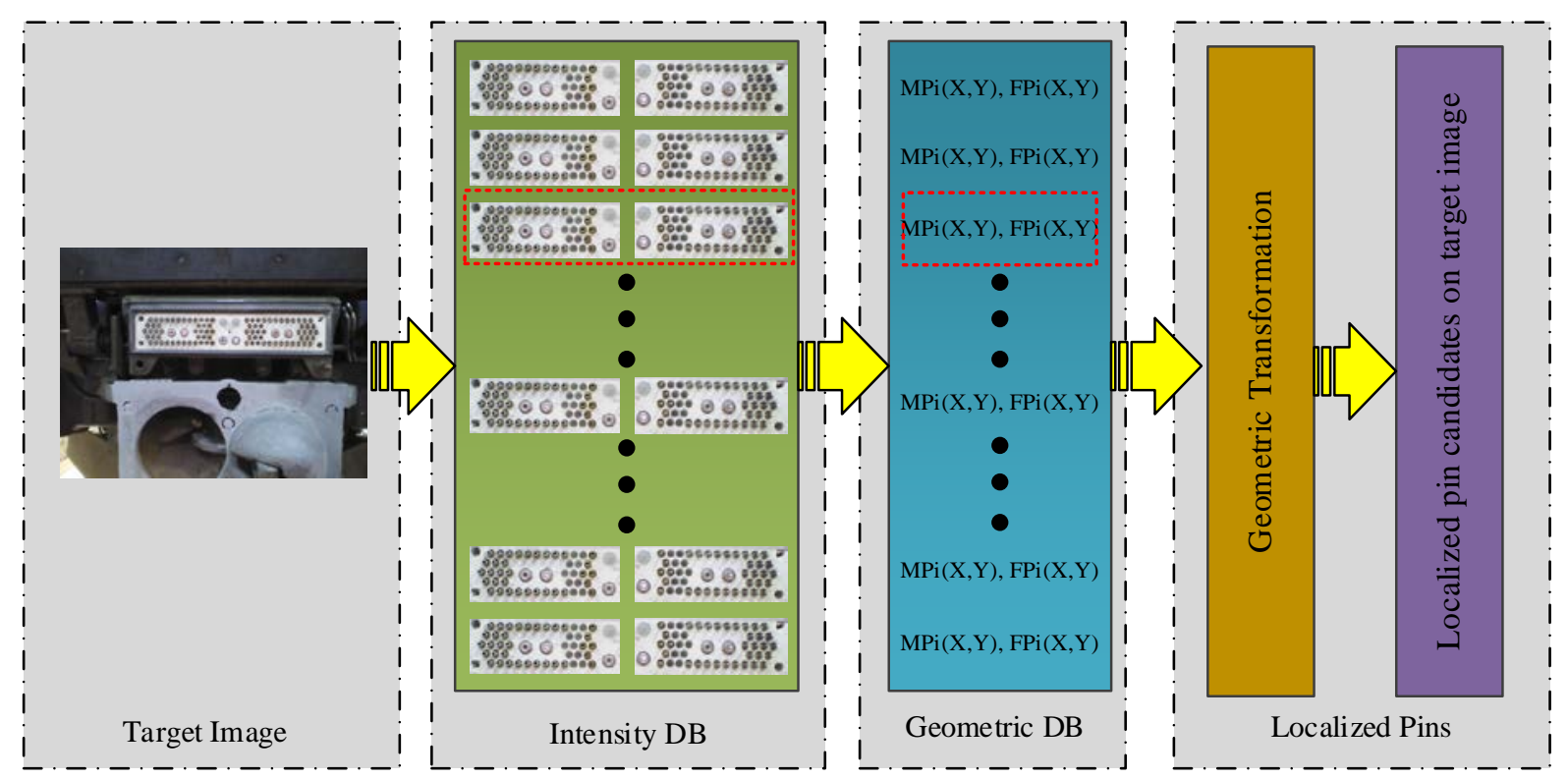

Figure 6: Summarized workflow of the VSMP feature extractor

The geometric information of electrical pins that are linked to the best-matched templates are then extracted and transformed from template to target image space. In order to accomplish this, the interest points in both target image and best-matched templates are identified using the Speeded Up Robust Features (SURF) point detector. The transformation corresponding to matched point pairs are estimated by employing M-estimator Sample Consensus (MSAC) algorithm, a variant of RANSAC which is robust to outliers.

The world coordinates of the pin candidates in the RGB camera frame are realised by the VSPM PE according to equation 4 . Here $\left[X_{i} Y_{i} Z_{i}\right]^{T}$ are the coordinates of the $i^{\text {th }}$ pin candidate $\left(P_{i}\right)$ in frame $\{\mathrm{C}\}, \mathrm{f}_{\mathrm{x}}, \mathrm{f}_{\mathrm{y}}$ are the focal length of RGB camera in pixels, $c_{x}, c_{y}$ are the principal point offset of RGB camera, $x_{i}, y_{i}$ are the image coordinates of $P_{i}$ in RGB image frame and $\delta_{\mathrm{x}}, \delta_{\mathrm{y}}$ are the distortion of RGB camera. The 2D to 3D correspondence of pinhole camera model is realised by the output of ToF camera, which provides the distance from camera to the target, hence the scale factor of RGB camera $\alpha$. Similar to motion planar in VSOM, the VSPM motion planner transforms $\left[\mathrm{X}_{\mathrm{i}} \mathrm{Y}_{\mathrm{i}} \mathrm{Z}_{\mathrm{i}}\right]^{\mathrm{T}}$ from camera frame to tool frame. 
$\left[\begin{array}{c}X_{i} \\ Y_{i} \\ Z_{i}\end{array}\right]=\left[\begin{array}{ccc}1 / f_{x} & 0 & -C_{x} / f_{x} \\ 0 & 1 / f_{y} & -C_{y} / f_{y} \\ 0 & 0 & 1\end{array}\right]\left[\begin{array}{c}\alpha\left(x_{i}+\delta_{x}\right) \\ \alpha\left(y_{i}+\delta_{y}\right) \\ \alpha\end{array}\right]$

\subsection{Force-position control module (FPCM) and voltage sensing module (VSM)}

\subsubsection{Hardware design}

The hardware of the FPCM consists of six force transducers which operate in pairs to measure forces along $X_{t}\left(F_{x}\right), Y_{t}\left(F_{y}\right)$ and $Z_{t}\left(F_{z}\right)$ - shown in figure 7. Each force transducer is coupled to the ATmega 2560 microcontroller via voltage amplifiers and 24-bit analogue-to-digital converters. The particular microcontroller was selected due to its capability to communicate with MALAB ${ }^{\circledR}$ Simulink ${ }^{\mathrm{TM}}$ in real-time. Two probes were designed such that 'Tool 1' is to inspect the Scharfenberg type railway couplers found in SIEMENS Class 380 rolling-stocks (also in majority of modern day rolling stock) and 'Tool 2' is to inspect "Voith" couplers found in SIEMENS Class 318 and 320 rolling stocks. It is important to note that only Tool 1, i.e. the Scharfenberg coupler inspection probe requires the force-position control module and included in this paper.

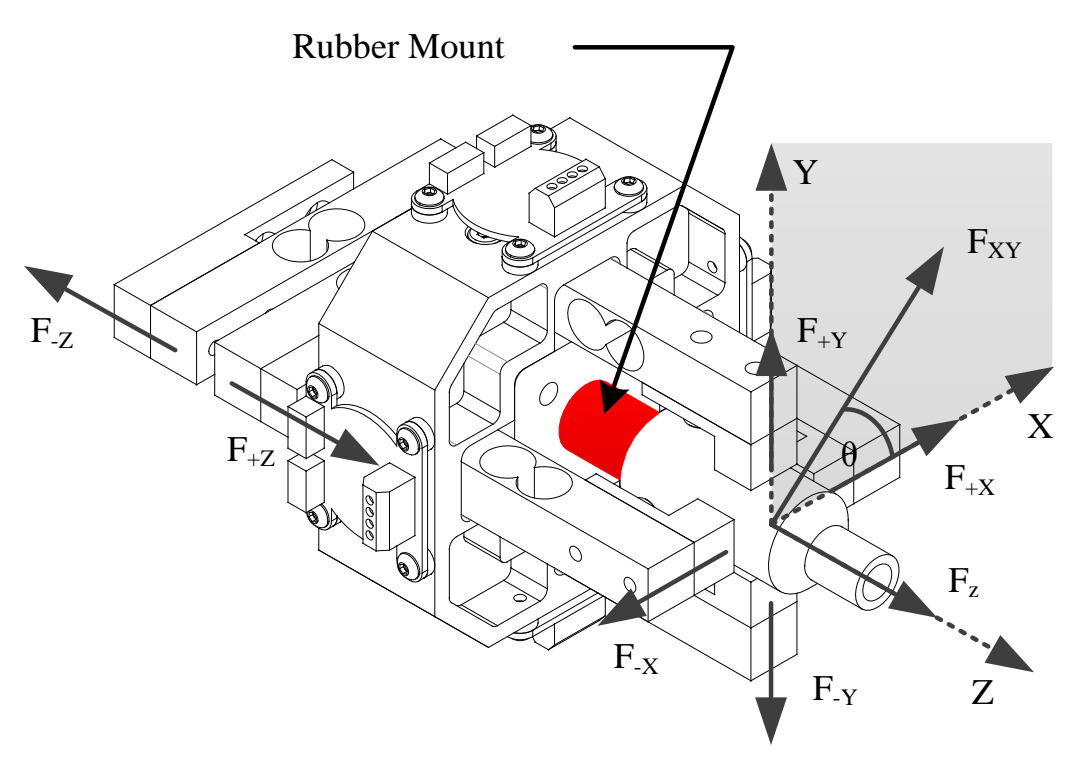

Figure 7: The key components of the force sensing structure, their physical arrangement and illustration of forces acting along each Cartesian axis in frame $\{\mathrm{T}\}$

The critical assembly tolerances of the EOAT are controlled at $\pm 0.05 \mathrm{~mm}$ and validated using a coordinate measurement machine. The Output of the VSPM motion planner acts as the input to the FPCM and therefore, the tip of EOAT is tapered conically with a predetermined angle such that it will partially compensate inaccuracies in VSPM output. In addition, it provides passive compliance for the initial engagement between a given pin and the EOAT, making the connection easier to locate. In addition, the internal structure of the probe consists of a spring-loaded tip such that the same probe could be used to examine both the male and the female pin candidates. Hence, the requirement to have multiple inspection 
probes or robotic tool changers is dismissed. The stiffness of the rubber mount was selected such that it provides adequate rigidity to return the tool tip to its initial position. Further, the rubber mount isolates the tool 'Tool 1' electrically from the rest of the metal structure.

\subsubsection{System modelling and controller design}

The FPCM controls the position of EOAT based on measured forces such that the desired engagement between the tool and a given pin is achieved. For instance, the FPCM extracts position $P_{i}$ from VSPM and jogs the robot to an approach position. The FPCM algorithm then increments the robot's position in $Z_{t}$ direction while tracking the resultant force and position along each Cartesian axis in frame $\{T\}$. Theoretically the resultant force in $X_{t} Y_{t}$ plane $F_{x y}=0$ during a smooth insertion and therefore, any $\left|F_{x y}\right|>0$ implies that the inner wall of tool rubs the electrical connector. The FPCM calculates new coordinates in frame $\{\mathrm{T}\}$ such that $\left|F_{x y}\right|=0$. However, in practice, and to reject noise and the effect of gravity on the sprung mass, a threshold $F_{r}(x y)$ greater than zero for $F_{x y}$ is opted. A complete insertion is realised when $F_{z}$ is greater than a threshold $F_{r_{z}}$. Once $F_{z} \geq F_{r}(Z)$ the FPCM will stop the operation and move to stage 4 where the VSM module takes over. Both the FPCM and the VSM execute alternatively for 68 iterations such that all electrical pins are examined.

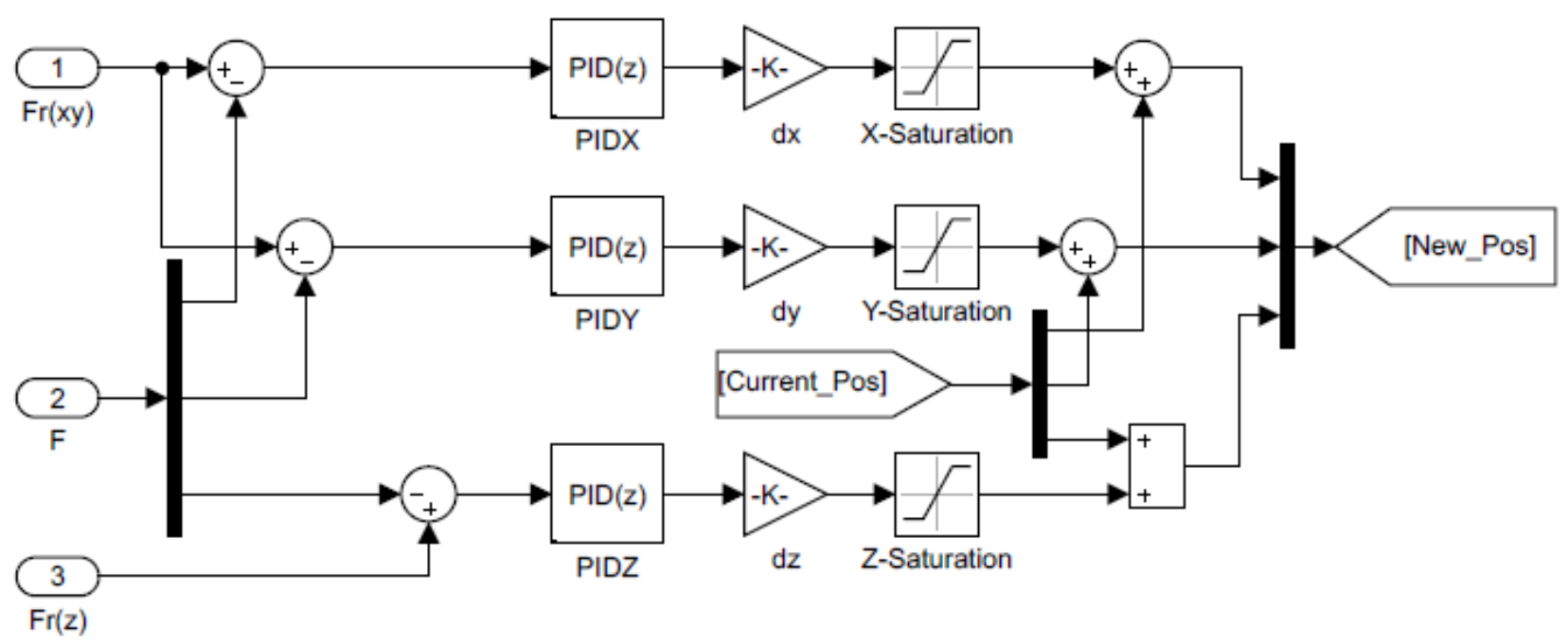

Figure 8: Cartesian force-position controller of the FPCM in frame $\{\mathrm{T}\}$

Three PID controllers were designed to control the force-potion along each Cartesian axis. For instance, consider the force-potion control along $X_{t}$ axis. Here it is assumed that a given force transducer obeys the Hooke's law within its operational range. Therefore, the measured resultant force $F$ can be expressed according to equation 5 where $k$ represents the spring constant and $x$ is the displacement of the force transducer. Further, the force tracking error $e$ can be written according to equation 6 . 
Equation 5

$F=k \cdot x$

Equation 6

$e=F_{r}-F$

The substitution of equation 5 in the equation 6 leads to below expression.

Equation 7

$e=F_{r}-(k \cdot x)$

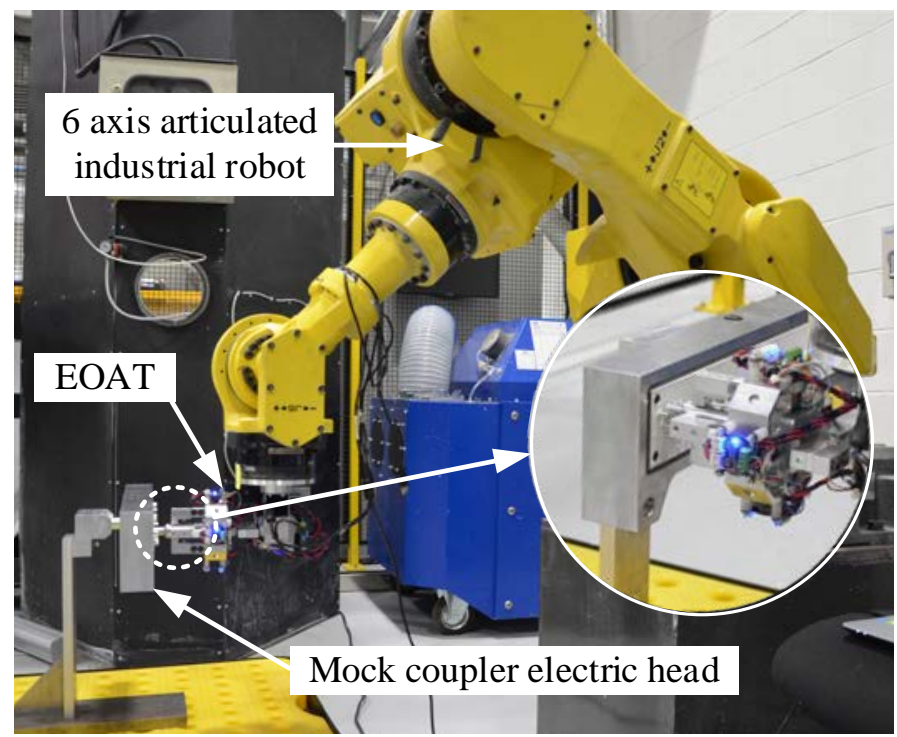

(a)

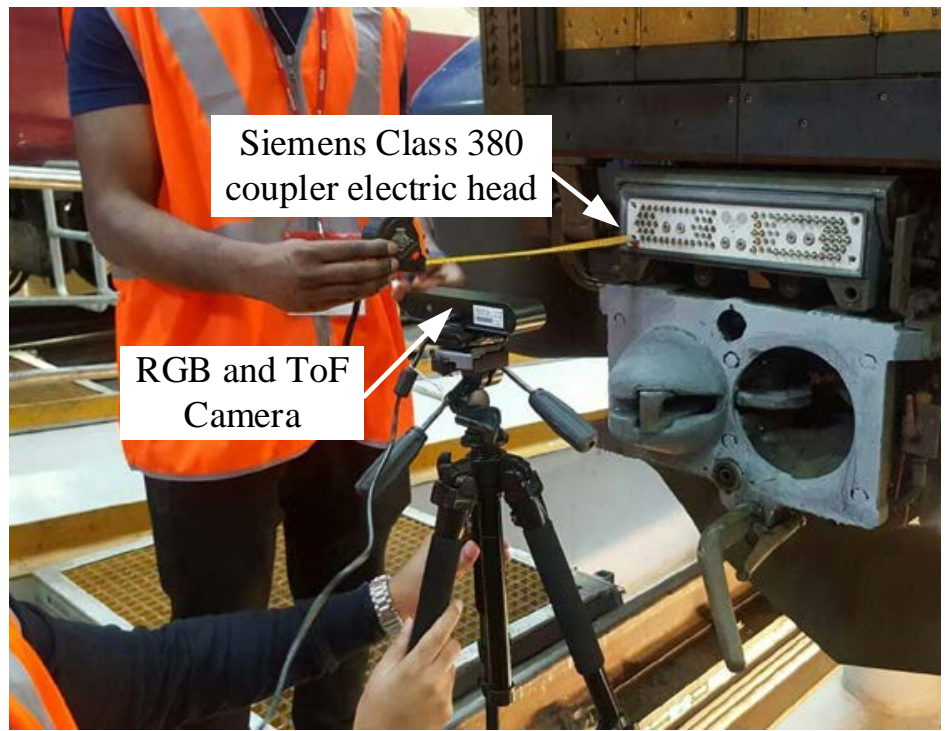

(b)

Figure 9: (a) Experimental setup using a commercial grade articulated 6-DoF robot and (b) data collection of the real Scharfenberg coupler using RGB and ToF cameras

Equation 8

$x=\frac{\left[F_{r}-\left(K_{p} e+K_{i} \int_{0}^{t} e d t+K_{d} \frac{d e}{d t}\right)\right]}{k}$

Equation 9

$K_{d} \frac{d^{2} e}{d t^{2}}+\left(K_{p}-1\right) \frac{d e}{d t}+K_{i} e=0$

In order to reduce the tracking error, the following PID controller in equation 8 is proposed. Here, $K_{p}, K_{i}$ and $K_{d}$ are the proportional, integral and derivative gains respectively. By substituting equation 8 in the 
equation 7 and taking its time derivative leads to equation 9, which is asymptotically stable for properly selected $K_{p}, K_{i}$ and $K_{d}$ values. Similarly, the force-position controllers in $Y_{t}$ and $Z_{t}$ directions were designed. Shown in figure 8 is the FPCM closed loop controller in all three directions. The constant $k$ was approximated to be in the range of $10^{-5} \mathrm{~N} / \mathrm{m}$ through force-displacement measurement using a 'Stable Microsystems' material tester. The maximum delta displacement of the controller output along a given axis has been bounded to $\pm 0.05 \mathrm{~mm}$ as a safety feature to avoid any overshoot of the EOAT.

\section{Experiments and results}

As a proof of the maintenance concept critical aspects of the system are evaluated in an experimental setup, which encompasses a commercial grade six axis Fanuc industrial robot and a mock railway coupler Figure 8 (a). However, performance of the system on actual coupler has been evaluated by collecting data of the real coupler -Figure 8 (b).

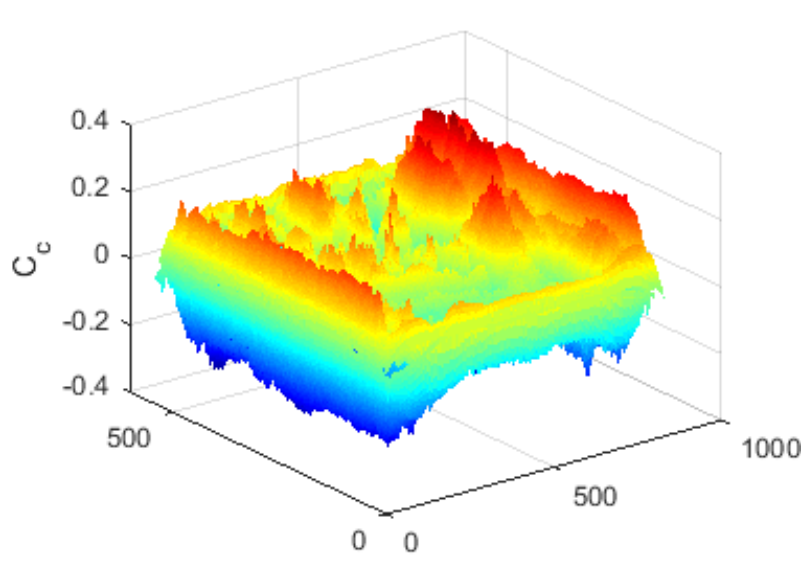

(a)

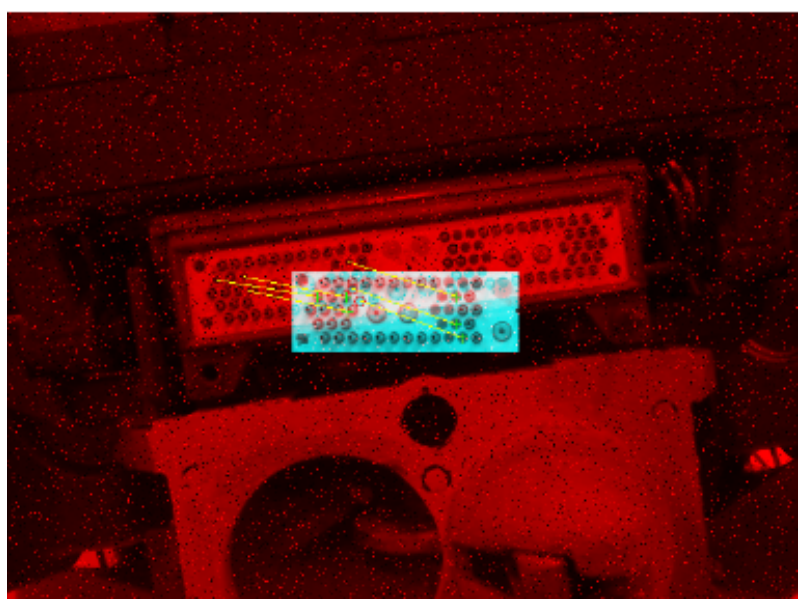

(c)

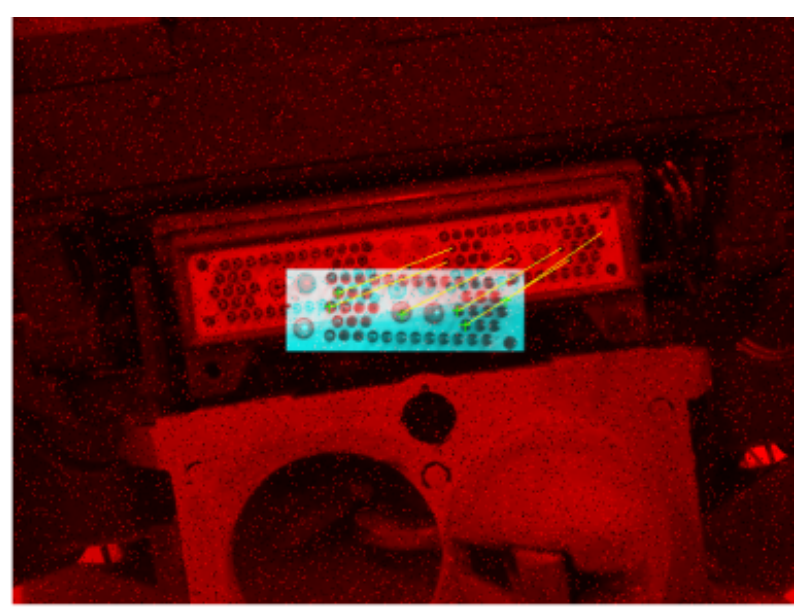

(b)

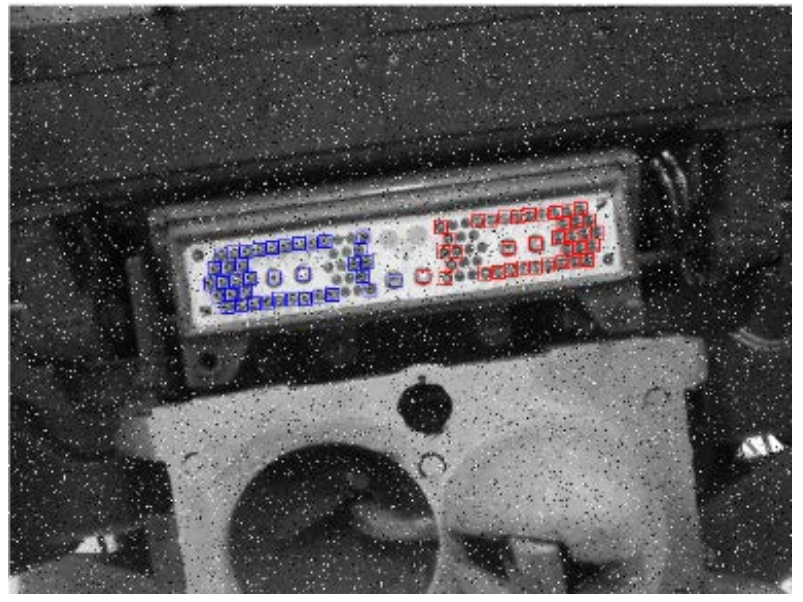

(d)

Figure 10: Performance of the VSPM feature detector on actual coupler: (a) $C_{c}$ of male and female templates, (b) matched points between target and male template images, (c) matched points between target and female template images, and (d) successfully localized connectors in image frame. 


\subsection{Performance of VSOM}

The performance of the VSOM is evaluated for a set of 31 random camera poses and 40 frames are captured in each pose at an approximated distance of $0.5 \pm 0.1 \mathrm{~m}$. The detection rate of VSOM FE is found to be $100 \%$. The performance of the VSOM PE is validated according to [18] by analysing the degree of fit of best-fit plane $D_{f}=\ln \left(\lambda_{3} / \lambda_{1}\right)$ where $\lambda_{1}$ is the smallest eigenvalue and $\lambda_{3}$ is the largest eigenvalue of the covariance matrix of coupler electric head facet 3D point cloud data, the sample mean and standard deviation of $D_{f}$ are 9.087 and 0.314 respectively. Furthermore, according to quantitative data provided in [18], it can be concluded that the accuracy of any fitted plane by VSOM is greater than $\pm 1^{0}$ since $9.0695<$ Mean $D_{f}<9.1046$. The, output of the VSOM therefore complies with anticipated tolerances and demonstrates capability of commercial implementation.

\subsection{Performance of VSPM}

The performance of the VSPM FE was validated on the real coupler and it has been identified that the algorithm is robust to existing lighting conditions, extreme rotation and noise - see Figure 9 where rotation, and artificial impulse noise are introduced to the image. Further, within 99\% confidence it has been estimated that the $1.08 \mathrm{~mm}<\mid$ Mean $\Delta E_{x}|<2.19 \mathrm{~mm}, 0.56 \mathrm{~mm}<|$ Mean $\Delta E_{y} \mid<2.63 \mathrm{~mm}$ and $0.38 \mathrm{~mm}<$ $\mid$ Mean $\Delta E_{z} \mid<1.01$ where $\Delta E_{x}, \Delta E_{y}$ and $\Delta E_{z}$ are the errors of VSPM PE in $X_{t}, Y_{t}$ and $Z_{t}$ directions respectively. Hence, the robustness and accuracy of VSPM FE and the PE are successfully validated prior implementation in the maintenance yard.

Table 1: Fine-tuned PID gains of individual controller

\begin{tabular}{|c|c|c|c|}
\hline Gains & X-Axis & Y-Axis & Z-Axis \\
\hline$K_{p}$ & 1.145 & 1.172 & 1.103 \\
\hline$K_{i}$ & 0.0196 & 0.0162 & 0.051 \\
\hline$K_{d}$ & 0.00121 & 0.00146 & 0.00208 \\
\hline
\end{tabular}

\subsection{Performance of FPCM}

The optimum values of $F_{r}(x y)$ and $F_{r}(z)$ were experimentally determined as $0.6 \mathrm{~N}$ and $4.7 \mathrm{~N}$ respectively. However, these values are increased by an approximated factor of 1.5 in order reject noises such as gravity, EOAT material/assembly elasticities and inaccuracies of the force transducers. For the safety PID gains are initially tuned offline by a dynamic modelling system in MATLAB ${ }^{\circledR}$ Simscape Multibody ${ }^{\mathrm{TM}}$ starting from relatively smaller values such that the EOAT will not overshoot in $X_{t} Y_{t}$ plane and damage the equipment. The fine-tuned PID gains through physical experiments are summarized in table 1. Here the PID tuner application incorporated with MATLAB ${ }^{\circledR}$ Control Systems Toolbox ${ }^{\mathrm{TM}}$ has been utilized. Average time 
consumed by the FPCM for a complete tool engagement is $3.972 S$ per pin which has been observed to depend on output accuracy of the VSPM. Shown in figure 11 (a) is the measured force and EOAT displacement in frame $\{\mathrm{T}\}$ when the Euclidean distance between TCP and pin centre $(D)$ in $X_{t} Y_{t}$ plane is $D=4.02 \mathrm{~mm}$ and insertion time $4.858 \mathrm{~S}$ and figure 11 (b) when $D=1.23 \mathrm{~mm}$ and insertion time is 3.689 $S$. Therefore, the average cycle time is expected to improve radically by introducing an artificial neural network to improve the error in VSPM PE.
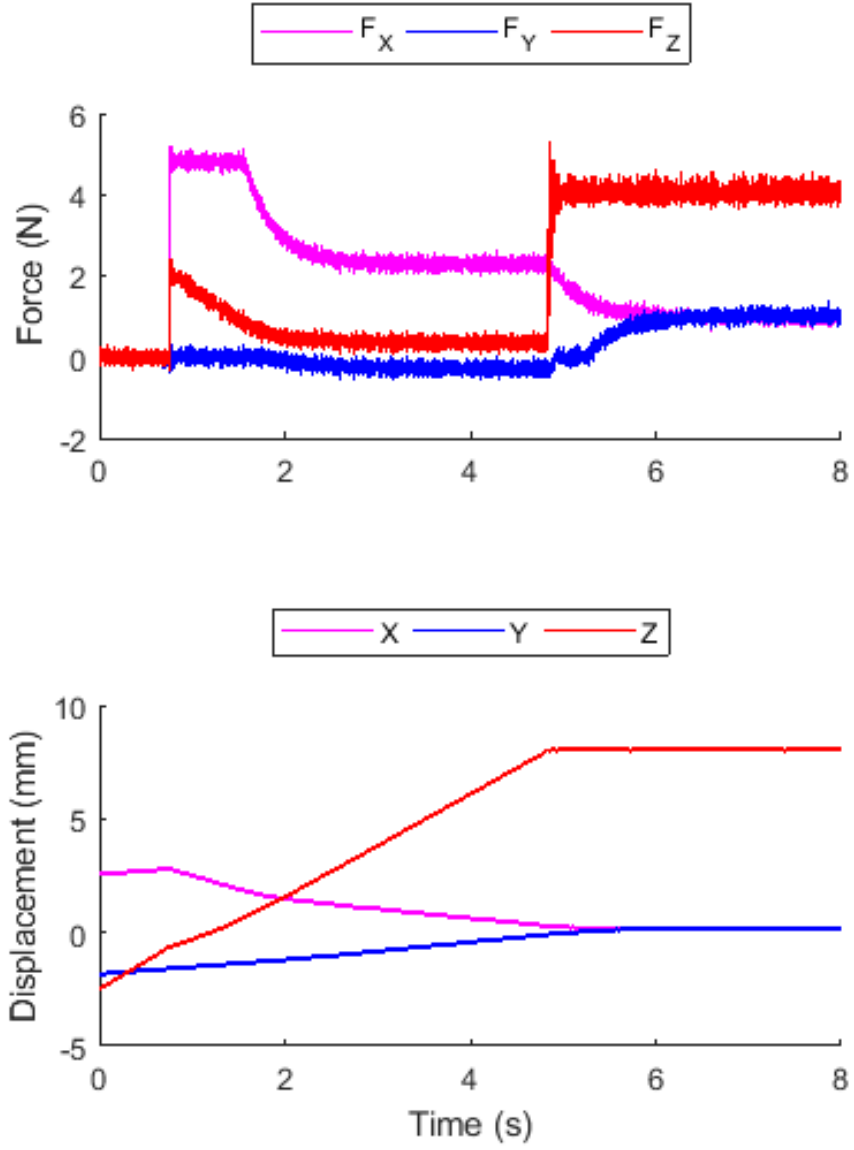

(a)
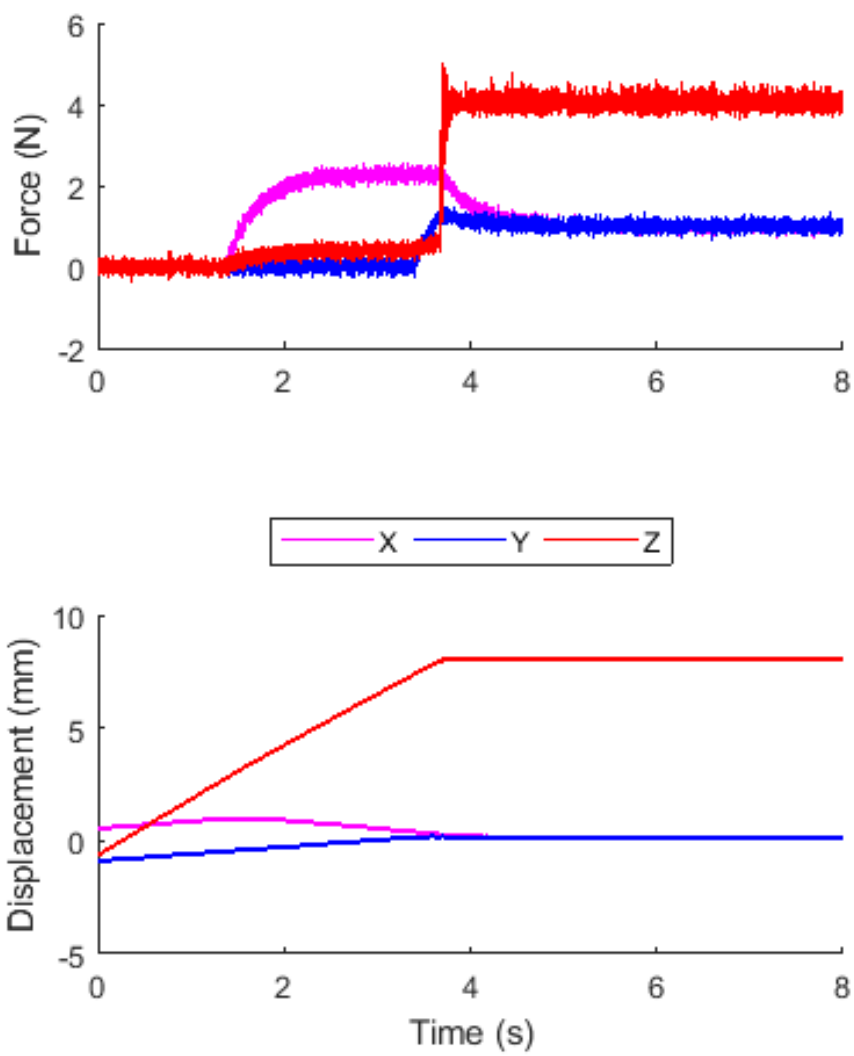

(b)

Figure 11: (a) Measured forces and displacement along each Cartesian axis in frame $\{\mathrm{T}\}$ when $D=4.02 \mathrm{~mm} \mathrm{~mm}$, and (b) measured forces and delta displacement along each Cartesian axis in frame $\{\mathrm{T}\}$ when $D=1.23 \mathrm{~mm} \mathrm{~mm}$

\section{Conclusion}

The proposed system demonstrates the feasibility of conducting automated inspection of Scharfenberg type railway coupler electric heads using industrial robots. Unlike some unified visual-force servoing techniques proposed in the literature, the system presented in this paper uses 3D vision for coarse orientation and positioning of the robot, and therefore, presents the opportunity to implement a low cost 3DoF force sensing structure. The system can be easily adapted to other type of couplers (or similar applications) by merely extending the VSPM-FE database. The, system can be an easy retrofit to most six 
DoF industrial robots once hand-eye and TCP calibration is performed. The prototyped robotic system is estimated to improve the standard operating time by 34\% approximately and with significantly enhanced reliability. The research will be extended to inspect mechanical and pneumatic components of the coupler in future.

\section{Acknowledgement}

This work is funded by Glasgow Caledonian University. Also, authors would like to thank Mr. Gary McLachlan of Abellio ScotRail, Glasgow, Mr. Roger Harris of Cyber-Weld Ltd, Mr. Tony Rodden and Mr. Alexandros Gkanatsios at Advanced Forming Research Centre, UK and Dr. Albert Nubiola of RoboDK. CA.

\section{References}

[1] T. Tomiyama, L. R. Garcıa, A. Kršlin, and G. Taykaldiranian, "Systems and conceptual design of a train cab front cleaning robot,” Procedia CIRP, vol. 59, pp. 61-66, 2017.

[2] L. Piln and G. Bissacco, "Development of on the machine process monitoring and control strategy in Robot Assisted Polishing,” CIRP Annals-Manufacturing Technology, vol. 64, no. 1, pp. 313-316, 2015.

[3] M. Farnsworth and T. Tomiyama, "Capturing, classification and concept generation for automated maintenance tasks,” CIRP Annals-Manufacturing Technology, vol. 63, no. 1, pp. 149-152, 2014.

[4] R. K. W. Vithanage, C. S. Harrison, and A. K. M. DeSilva, "A Study on Automating Rolling-stock Maintenance in the Rail Industry using Robotics," in Proceedings of the 14th International Conference on Informatics in Control, Automation and Robotics - Volume 2: ICINCO,, 2017, pp. 278283.

[5] M. Agheli, L. Qu, and S. S. Nestinger, "SHeRo: Scalable hexapod robot for maintenance, repair, and operations,” Robotics and Computer-Integrated Manufacturing, vol. 30, no. 5, pp. 478-488, 2014.

[6] E. Gilabert and A. Arnaiz, "Intelligent automation systems for predictive maintenance: A case study," Robotics and Computer-Integrated Manufacturing, vol. 22, no. 5-6, pp. 543-549, 2006.

[7] D. Hong, S. A. Velinsky, and K. Yamazaki, "Tethered mobile robot for automating highway maintenance operations,” Robotics and Computer-Integrated Manufacturing, vol. 13, no. 4, pp. 297307, 1997.

[8] Y. J. Kwon, R. Chiou, and L. Stepanskly, "Remote, condition-based maintenance for web-enabled robotic system,” Robotics and computer-integrated manufacturing, vol. 25, no. 3, pp. 552-559, 2009.

[9] C. Mineo, S. G. Pierce, P. I. Nicholson, and I. Cooper, "Robotic path planning for non-destructive testing-A custom MATLAB toolbox approach," Robotics and Computer-Integrated Manufacturing, vol. 37, pp. 1-12, 2016.

[10] G. Reinhart and W. Tekouo, "Automatic programming of robot-mounted 3D optical scanning devices to easily measure parts in high-variant assembly,” CIRP Annals-Manufacturing Technology, vol. 58, no. 1, pp. 25-28, 2009.

[11] G. Horváth and G. Erdős, "Point cloud based robot cell calibration," CIRP Annals-Manufacturing Technology, 2017.

[12] L. Caruso, R. Russo, and S. Savino, "Microsoft Kinect V2 vision system in a manufacturing application,” Robotics and Computer-Integrated Manufacturing, vol. 48, pp. 174-181, 2017.

[13] P. Rea and E. Ottaviano, "Design and development of an Inspection Robotic System for indoor applications,” Robotics and Computer-Integrated Manufacturing, vol. 49, pp. 143-151, 2018. 
[14] A. E. K. Mohammad, J. Hong, and D. Wang, "Design of a force-controlled end-effector with lowinertia effect for robotic polishing using macro-mini robot approach,” Robotics and ComputerIntegrated Manufacturing, vol. 49, pp. 54-65, 2018.

[15] P. Schumacher and M. Jouaneh, “A force sensing tool for disassembly operations,” Robotics and Computer-Integrated Manufacturing, vol. 30, no. 2, pp. 206-217, 2014.

[16] D. J. Agravante, A. Cherubini, A. Bussy, P. Gergondet, and A. Kheddar, "Collaborative humanhumanoid carrying using vision and haptic sensing," in Robotics and Automation (ICRA), 2014 IEEE International Conference on, 2014, pp. 607-612.

[17] K. Hormann and A. Agathos, "The point in polygon problem for arbitrary polygons," Computational Geometry, vol. 20, no. 3, pp. 131-144, 2001.

[18] O. Fernández, “Obtaining a best fitting plane through 3D georeferenced data,” Journal of Structural Geology, vol. 27, no. 5, pp. 855-858, 2005. 